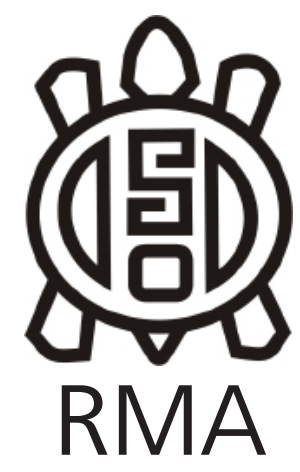

Museología

\title{
Aprendizajes y percepciones en la Noche de los Museos
}

\author{
Learning and perceptions at Museum Night
}

\author{
María Fernanda Melgar*, Romina Cecilia Elisondo**, \\ Florencia E. Fernández ${ }^{\star \star *}$ y Lazarte. D. L. Vanesa****
}

*Facultad de Ciencias Humanas. Universidad Nacional de Río Cuarto. E-mail: fernandamelgar@gmail.com

**Facultad de Ciencias Humanas. Universidad Nacional de Río Cuarto. E-mail: relsiondo@gmail.com

***Facultad de Ciencias Humanas. Universidad Nacional de Río Cuarto. E-mail: flor_fernandez_07@hotmail.com

****Facultad de Ciencias Humanas. Universidad Nacional de Río Cuarto. E-mail:vanesalazarte28@gmail.com

\begin{abstract}
Resumen
La Noche de los Museos es un evento internacional que interpela a nuevos públicos. En el artículo presentamos un estudio cualitativo sobre percepciones de diferentes grupos respecto del evento. Nos proponemos comprender percepciones y valoraciones respecto del evento construidas por personas que participaron en la NM Río Cuarto ediciones 2017 y 2018. Nos interesa en esta investigación focalizar en los procesos de aprendizaje desarrollados por los participantes en el marco del evento. Como instrumentos de recolección de datos se utilizaron cuestionarios y entrevistas. Para enterarse del evento el medio más empleado es el "boca en boca" y algunas redes sociales. La mayoría de las personas mencionaron que concurren al evento porque les interesa explorar y conocer cosas nuevas. Los amigos, la familia y la pareja, son los grupos que más se destacaron para compartir el evento. La diversidad de actividades y contenidos fueron lo que más les gustó a los públicos. Los análisis emergentes resultan de interés para el diseño de políticas educativas y culturales referidas a la NM.
\end{abstract}

Palabras claves: Noche de los Museos; Aprendizaje; Contexto; Museos; Educación.

\begin{abstract}
Museum Night is an international event that invite new audiences. In the article we present a qualitative study on perceptions of different groups regarding the event. We propose to understand perceptions and evaluations regarding the event built by people who participated in the NM Río Cuarto editions 2017 and 2018. We are interested in this research to focus on the learning processes developed by the participants within the framework of the event. Questionnaires and interviews were used as data collection instruments. To find out about the event, the most widely used means is mouth to mouth and some social networks. Most of the people mentioned that they attend the event because they are interested in exploring and knowing new things. Friends, family and couples are the groups that stood out the most to share the event. The diversity of activities and contents were what the public liked the most. The emerging analyzes are of interest for the design of educational and cultural policies related to NM.
\end{abstract}

Keywords. Museum Night; Learning; Learning context; Museum; Education.

Los museos buscan acercarse a públicos diversos, abren sus puertas y orientan sus acciones hacia grupos cada vez más amplios y heterogéneos. Todo lo que sucede en los museos, de alguna manera, se vincula con enseñar y aprender a partir de interacciones entre grupos, objetos e historias. Es decir, la finalidad de los museos, en tanto contextos educativos no formales, es generar espacios para las experiencias de aprendizaje culturales.
La Noche de los Museos (NM en adelante) es un claro ejemplo de estas instituciones por abrirse a nuevos públicos, generar experiencias significativas de aprendizaje y propiciar vínculos novedosos entre personas, historias y expresiones culturales. El evento la Noche de los Museos surgió en la ciudad de Berlín, Alemania en 1977, donde se realizó por primera vez a nivel mundial. El mismo fue denominado "Larga Noche de los Museos y fue 
imitado por otras ciudades de Alemania y 150 países del mundo. Durante la segunda mitad del siglo XX los museos comienzan a cambiar su concepción, de estar centrados en los objetos a prestar atención a los sujetos y a los públicos. El evento la NM consiste en que una vez al año los museos abren sus puertas al público en general en horario nocturno, brindando la posibilidad de poder conocer dichas instituciones. En América, el primer lugar donde se realizó la NM fue en Argentina, en la ciudad de Buenos Aires en el año 2004 (Elías y Leonardi, 2018). El evento, con el correr de los años empezó a cobrar más fuerzas y relevancia, logrando llegar a ciudades como Alta Gracia, Córdoba (Zabala y De Carli 2015) Santa Fe, La Plata, Rosario, Ushuaia y Río Grande (Elías y Leonardi, 2018).

En la ciudad de Río Cuarto, el evento NM se realiza de manera ininterrumpida desde 2014. La primera edición se denominó La Noche de los Museos a la Luz de la Luna, organizada Subsecretaría de Cultura del Municipio de Río Cuarto, junto a museos, salas y distintas instituciones de la ciudad. En la segunda edición se amplió la oferta cultural y educativa a más de 15 espacios culturales, museos, salas e instituciones. Durante el evento se realizaron actividades en las calles de la ciudad: recitales, visitas guiadas, talleres, representaciones teatrales, etc. (Melgar, Elisondo y Chiecher, 2016). En 2016 se llevó a cabo la tercera edición del evento en Río Cuarto, incluyendo actividades de artes visuales, cine, tango, folclore, música en vivo, fotografía, arte textil, cerámica, almacén de arte y propuestas de los talleres de arte y cultura de la UNRC (Melgar, Chiecher, Elisondo y Donolo (2017). Durante la cuarta (2017) y quinta edición (2018) se continuó ampliando la oferta cultural y los lugares de participación. La UNRC siguió teniendo un papel activo a través de la comunicación de diferentes propuestas ofrecidas en el marco del Departamento de Artes. Pintura, fotografía, cerámica, música, danza, teatro y recorridos guiados por los museos, fueron algunas de las actividades. Otro dato distintivo de estas ediciones fue la participación de espacios educativos formales de enseñanza superior y nivel medio. Como elemento distinto, en 2018 , se ofrecía un mapa con la ubicación de los diferentes puntos y actividades, aspecto que permitía a los públicos una organización de sus recorridos. En la sexta edición (2019), el evento se desarrolló con la participación de diferentes espacios en los que se realizaron alrededor de 80 actividades. Un aspecto novedoso para esta edición fue la apertura del Observatorio del ex Colegio Nacional. Esta actividad, resultó innovadora porque además se realizó de manera conjunta con el Observatorio de la ciudad de Córdoba, implicó la posibilidad de contemplar el cielo con un telescopio y realizar múltiples actividades interactivas de ciencia.

En el presente estudio nos proponemos comprender percepciones y valoraciones respecto del evento construidas por personas que participaron en la NM Río Cuarto ediciones 2017 y 2018. Nos interesa en esta investigación focalizar en los procesos de aprendizaje desarrollados por los participantes en el marco del evento. Además, nos propusimos comprender desde la perspectiva de los sujetos el contexto social y motivacional de la experiencia. Consideramos que la investigación puede construir significativamente en el diseño de políticas y programas educativos vinculados a la NM y a otros eventos en museos y espacios culturales. Los datos emergentes en el estudio resultan de relevancia para comprender, desde las voces de los participantes, particularidades de la experiencia y de los aprendizajes construidos.

El artículo se estructura de la siguiente manera: en primer lugar, presentamos estudios actuales sobre la NM en Argentina, luego nos referimos a aspectos metodológicos de la investigación y analizamos los resultados a partir de la construcción de seis categorías:1) ¿Quiénes? Los públicos en la Noche de los Museos; 2) ¿Cómo te enteraste? Comunicación de la NM; 3) ¿Con quién y por qué? NM como experiencia social y motivacional; 4) ¿Que te gustó? Aspectos destacados en la NM; 5) ¿Qué te gustaría? El público propone y 6) ¿Aprender en la NM? Por último, presentamos consideraciones finales y futuras líneas de investigación e intervención.

\section{Estudios en la Argentina sobre la Noche de los Museos}

En la última década, en el contexto internacional, se han realizado estudios en diferentes países sobre la NM. En general, las investigaciones indican que el contexto nocturno de la visita crea una relación diferente con el museo que posibilita nuevas exploraciones y vínculos entre las personas y las instituciones (Germain, 2016). Asimismo, tal como lo destacan Barron y Leask (2017), la NM atrae a públicos jóvenes que prefieren las actividades nocturnas. Gordin y Dedova(2014) exploraron las dimensiones de creatividad del evento de la Noche de los Museos celebrado en San Petersburgo en 2013. Los resultados revelaron que el evento afecta el comportamiento de los visitantes y conduce a cambios en la estructura del público objetivo. Al organizar el evento, las instituciones culturales utilizan soluciones innovadoras que aplican un enfoque más creativo hacia las actividades organizadas y los visitantes. Esto, a su vez, fomenta el desarrollo positivo de la red de comunicación interna en las instituciones culturales. Además, el evento promueve la visita a museos que no suelen ser muy frecuentados, y que se podría decir, se encuentran a la sombra de los museos más reconocidos. Según los autores, la sensibilización, la creación de una imagen positiva y la atracción de visitantes a la gran cantidad de museos de San Petersburgo son funciones clave del evento.

En Argentina también se han realizado interesantes investigaciones respecto de la NM como evento cultural y educativo. Bialogorski y Fritz (2004) realizaron una investigación en seis museos la Ciudad de Buenos Aires. Para la recolección de datos se entrevistaron a 129 participantes de la NM. Los resultados indican que la noche de los museos despierta el espíritu de comunidad 
y promueve el uso del espacio público. Respecto de las motivaciones, las personas destacan el deseo de conocimiento, la nocturnidad y gratuidad del evento. Según las autoras, se observa una tendencia de los visitantes a constituir la Noche de los Museos como un hábito cultural. Además, destacan el importante impacto educativo del evento como construcción comunitaria para el uso del espacio público y del patrimonio compartido.

Zabala y De Carli (2015) sistematizaron las opiniones y vivencias que tienen los visitantes del Museo de Antropología de la Facultad de Filosofía y Humanidades de la Universidad Nacional de Córdoba en la NM 2012. Los resultados indicaron que los participantes construyen una significación especial de la visita en tanto propuesta nocturna, compartida con amigos y familiares, que posibilita el conocimiento de nuevos espacios culturales y educativos. Las autoras concluyen que la propuesta cultural de la Noche de los Museos es parte del proceso de comunicación entre los museos y la sociedad, generando una apropiación de esos espacios que aún para muchos visitantes sigue siendo el lugar de un sector social privilegiado y distinguido.

Elías y Leonardi (2018) realizaron una investigación, en Ciudad Autónoma de Buenos Aires durante 2017, para analizar la evolución, el impacto socioeconómico y cultural de la NM. Se utilizó una encuesta mixta, diseñada en los formularios de Google. Se hizo una revisión de las conceptualizaciones de turismo cultural y de museos, de las valoraciones del evento y su impacto. La muestra fue de carácter no probabilístico. Se utilizó una encuesta distribuida vía mail y redes sociales a 265 personas. Se basó en un análisis exploratorio-descriptivo. Los resultados, se subdividieron en: la caracterización sociodemográfica de los encuestados; examinación e interpretación de las preguntas que se focalizan en la NM y su valoración; y finalmente, la consideración respecto al impacto que tuvo el evento. Los resultados expresaron que la mayoría de las personas que asistieron al evento fueron de género femenino entre 25 y 35 años con un nivel educativo alto. El 50\% de los que participaron del evento, habían visitado el evento anteriormente. Las personas tuvieron conocimiento del evento, en primer lugar, a través de las redes sociales, segundo, por el "boca en boca". En cuanto a los medios de transporte se destacó el automóvil particular, luego caminando y, por último, los colectivos. En relación al tiempo que las personas destinan al evento, el mayor porcentaje le dedicó entre una y tres horas. Ante la pregunta ¿cómo evaluaría el evento? la mayoría lo calificaba como muy bueno. En cuanto al impacto de la NM el $98 \%$ de las personas consideraban que el evento contribuyó a difundir el arte y la cultura, mientras que el $8 \%$ supone importante el impacto del evento sobre la actividad económica de la ciudad.

En la ciudad de Córdoba, en el marco de un trabajo final de licenciatura en Psicología Milman (2018) desarrolló un estudio cuyo objetivo fue la comprensión de las significaciones de la NM por parte de los visitantes y de los organizadores, la descripción y análisis de las preferencias por los cuales los visitantes decidieron ir al museo, las expectativas y vivencias. Además, realizó un aporte a la construcción del rol del psicólogo dentro del museo. Como resultado se obtuvo que las personas deciden participar del evento porque se desarrolla de noche y se facilita el transporte. Con respecto al rol del psicólogo se realizó un trabajo desde la psicología cognitiva conductual vinculado a museos.

En la ciudad de Río Cuarto se han realizado dos estudios preliminares sobre la NM. En el primero, Melgar, Elisondo y Chiecher (2016) presentan un estudio de público de carácter exploratorio realizado con sujetos que participaron de La Noche de los Museos en 2015. Entre los resultados se destacan motivaciones vinculadas a la curiosidad, la posibilidad de participar en el horario nocturno, experiencias previas e interés por conocer el museo. Las autoras concluyen que la NM es una iniciativa que promueve la creatividad, las experiencias compartidas y los vínculos entre museos y sociedad. El segundo estudio realizado en Río Cuarto tuvo como objetivo conocer cuáles son las percepciones y experiencias que el público tiene en la NM. Se seleccionaron tres museos de la ciudad de Río Cuarto; el Museo Histórico Regional, el Museo Tecnológico Aeroespacial y el Museo Municipal de Bellas Artes. Para la recolección de datos se utilizó un cuestionario contenía ítems abiertos y cerrados. El total del público encuestado fue de 120 personas. En cuanto a los resultados sostuvo que la NM es un evento que lleva un público muy diverso y que deciden participar por diferentes motivos, el evento es significado por parte de las personas que asisten como un espacio, un ambiente dónde se puede compartir, conocer, vivenciar una nueva oportunidad, para participar de un espacio público, una fiesta, una determinada cultura, etc. (Melgar, Chiecher, Elisondo y Donolo, 2017).

En suma, los estudios, aunque se han realizado en diferentes contextos, indican ciertas representaciones compartidas de los participantes sobre NM. El evento se constituye en una oportunidad de acercamiento entre ciertos públicos y los museos, especialmente públicos que no visitan asiduamente estas instituciones. Las personas participan motivadas por el deseo de conocer y compartir una experiencia novedosa con amigos y familiares. Asimismo, los participantes destacan el espíritu de comunidad del evento y el carácter nocturno y gratuito del mismo.

\section{Consideraciones metodológicas}

Se realizó un estudio desde un enfoque cualitativo para conocer las diferentes percepciones y aprendizajes que construyen las personas en la NM. Se conformó una muestra de tipo no probabilística casual. Participaron del estudio 172 personas que asistieron a la NM en los siguientes museos de Río Cuarto: Centro Cultural Trapalanda, Museo Histórico Regional, Tintorería Japonesa, Casa de la Cultura, y Museo del Riel. La recolección de datos se realizó en 2017 ( $N=30)$ y en $2018(\mathrm{~N}=142)$. 
Para la recolección de datos se emplearon cuestionarios y entrevistas semi-estructuradas. El cuestionario estaba organizado en apartados compuestos de ítems con respuestas abiertas y cerradas. En el primer apartado se solicitaba datos personales y el museo en el cual se administró el instrumento. El segundo, indagaba sobre el medio por el cual la persona se enteró del evento. En el tercer apartado, se preguntó con quién asistió al evento. El cuarto permitió conocer los motivos de la participación. El quinto, preguntaba acerca de lo que más les gustó. El sexto apartado, consistió en indagar si la persona modificaría algún aspecto del evento. El séptimo apartado refería a si reconocía alguna mejora en el evento, en caso de haber asistido en años anteriores. El siguiente, indagaba sobre el medio de transporte utilizado para llegar al evento y el último, interrogó respecto de la posibilidad de realizar alguna modificación, proponiendo alguna otra actividad o sugerencia para el próximo evento. Otro dato relevante fue que se dejó un espacio para que las personas, si lo deseaban, brindarán alguna información de contacto para poder comunicarnos.

Las entrevistas se realizaron con el objetivo de conocer con mayor profundidad las experiencias de algunos sujetos durante las ediciones de 2017 y 2018 de la NM. A partir del análisis de los datos emergentes de los cuestionarios, se seleccionaron diez sujetos de ambos años. Cada entrevista se conformaba por preguntas como las siguientes; ¿qué recordás del evento?, ¿qué sugerencias y aportes podrías proponer acerca del mismo?, ¿qué te gustaría que pasara en el evento?, ¿qué recuerdas acerca del público que asistió esa noche? y ¿cuáles fueron las razones que te llevaron a asistir al evento y por qué a ese museo?

En el análisis, se codificaron de manera abierta los datos, se triangularon resultados y se construyeron las categorías y subcategorías mediante comparaciones constantes. La investigación se desarrolló conforme a pautas y normas éticas definidas por organismos internacionales: se solicitó el consentimiento informado, se preservó el anonimato y la confidencialidad de los datos.

\section{Resultados}

¿Quiénes? Los públicos en la Noche de los Museos Los datos recolectados indican una mayor participación de mujeres (70\%) en el estudio. Estos resultados se corresponden con los hallados en estudios previos (Melgar et al, 2016 y 2017; Zabala y De Carli (2015) en los cuales también se observa mayor participación de mujeres.

De acuerdo a la edad, en general, en ambos años asistió un público diverso, si bien hay algunos grupos que se destacan más, existe un importante número de visitantes en cada franja etaria. Siendo la mayoría de las personas que asistieron al evento un grupo de niños, adolescentes y adultos jóvenes. Encontramos características similares, en el estudio de Melgar et al. (2017) en el cual se encontró un público diverso, con una franja etaria amplia, desde los 10 hasta más de 60 años. Por otro lado, el estudio de Bialogorski y Fritz (2004) en la NM en Buenos Aires los participantes tenían entre 36 y 55 años, por lo que lo calificaron como un público de jóvenes y adultos jóvenes. Zabala y De Carli (2015) pudieron observar que un porcentaje de los sujetos encuestados (30\%) eran estudiantes.

Según la ocupación de los participantes, se pudo observar lo siguiente: estudiantes (56 personas), profesionales $(\mathrm{N}=46)$, empleados $(\mathrm{N}=18)$, profesionales vinculados al arte $(\mathrm{N}=10)$, desempleados $(\mathrm{N}=9)$, jubilados $(\mathrm{N}=9)$ y amas de casa $(\mathrm{N}=5)$. Podemos indicar que el tipo de público que más se destacó en la NM de ambos años han sido los estudiantes, personas jóvenes que se encuentran en su período de formación académica, ya sea básica o universitaria. El estudio realizado por Melgar et al. (2017) se pudo apreciar que el tipo de público que asistía a este tipo de evento eran mayormente estudiantes, seguido de la ocupación profesionales y en tercer lugar empleados. Por otra parte, los autores Zabala y De Carli (2015) coinciden con estos resultados, obteniendo en su investigación una mayor participación en el evento del grupo de estudiantes.

\section{¿Cómo te enteraste? Comunicación en la NM}

Los principales medios por los cuales las personas se enteraron del evento en 2017 y 2018 fueron "de boca en boca" ( $\mathrm{N}=107)$, Facebook $(\mathrm{N}=58)$, medios masivos clásicos de comunicación: radios, televisión y diario $(\mathrm{N}=36$ personas); medios impresos o gráficos: afiches/folletos y la guía cultural ( $\mathrm{N}=28$ personas); WhatsApp $(\mathrm{N}=17)$; Twitter e Instagram ( $\mathrm{N}=9)$. El medio más empleado en las dos ediciones por las personas para enterarse del evento fue el "boca en boca", seguido de las redes sociales, específicamente, el facepage del evento. Estos resultados se dieron de igual manera en ambos años. Bialogorski y Fritz (2004) obtuvieron en su artículo que los medios de comunicación más renombrados fueron, el "boca en boca", seguido de la opción de "varios medios" y, por último, la opción de internet. Por otro lado, Melgar et al. (2017) obtuvieron que la red social facebook, específicamente del evento, fue el medio indicado y efectivo para dar a conocer el evento. En segundo lugar, se posicionaba el "boca en boca" como medio de difusión. Los resultados del estudio coinciden con los hallados por Zabala y De Carli (2015), tanto internet como el "boca en boca", fueron los medios de difusión más nombrados y con mayor efectividad desde la perspectiva de los encuestados. Los medios digitales van ganando cada vez más relevancia y efectividad al momento de realizar la difusión de algo en particular, en este caso el evento de la NM, si bien son importantes los folletos, afiches y guías culturales, la mayoría de los ciudadanos poseen algún elemento tecnológico (celular, computadora, tablets) por dónde esta información digitalizada le puede llegar de manera instantánea, masiva y económica.

\section{¿Con quién y por qué? NM como experiencia social y} motivacional

En relación a los aspectos sociales, identificamos que 
la mayoría de las personas asistieron acompañados por alguien: con amigos $(\mathrm{N}=65)$, con la familia $(\mathrm{N}=42)$, con su pareja $(\mathrm{N}=34)$, con compañeros de trabajo $(\mathrm{N}=2)$. También hay personas que van solas, pero en menor medida $(\mathrm{N}=28)$. El evento aparece, así como una instancia para compartir con otros. En comparación con los resultados de otras investigaciones se pudo indicar que de acuerdo a las autoras Melgar et al. (2017) el grupo predominante para asistir a este tipo de evento era la familia, luego los amigos, en tercer lugar, se encuentra el grupo de la pareja; y, por último, solos. En otro artículo realizado anteriormente por Melgar et al. (2016) se pudo observar como resultado que las personas prefirieron asistir a este tipo de eventos con su familia, conformando dicho resultado el $62 \%$ de la muestra, seguido de la opción amigos y, por último, la elección de visitarlo de manera individual.

El evento de la NM se convierte en un espacio ideal para compartir nuevas experiencias y vivencias ya sea acompañado por la familia, los amigos, la pareja, o por qué no también, solos/as, como un momento de conexión con nuestro ser, nuestra persona, ver que sentimientos y emociones generan esa diversidad de cultura en nosotros.

En relación a los aspectos que motivan la participación de las personas en el evento se destaca el interés por manifestaciones artísticas y por conocer y explorar diferentes aspectos del museo. En este sentido, a modo de ejemplo compartimos las palabras de algunas personas: "me dio curiosidad", "conocía del evento, pero nunca participé", "me gustan los museos y no puedo visitarlos en los horarios habituales porque trabajo", "fui al evento en otras ciudades y quería ver cómo era en la mía", "quería compartir con amigos/ familial pareja una actividad distinta en el tiempo libre".

Bialogorski y Fritz (2004) indicaron que el público asistía al evento por tres razones: la nocturnidad, la gratuidad y el deseo de conocimiento. Los participantes manifestaron afán de conocimiento, motivado por la curiosidad o algún interés específico referido a temáticas propuestas por los museos. En Melgar et al (2016) también se observamos que muchas personas expresan haber participado por curiosidad.

Cuando los museos ofrecen actividades que salen de las rutinas esperadas por los visitantes, parecen actuar como disparadores de la curiosidad (...) La curiosidad aparece relacionada con la creatividad, y en este sentido, cuando hablamos de museos como contextos creativos, destacamos su rol de ofrecer experiencias diversas a los visitantes que activen su curiosidad. (Melgar et al., 2016: 45)

¿Qué te gustó? Aspectos destacados en la Noche de los Museos

La mayoría de los participantes manifestó su agrado por la diversidad de actividades que los espacios culturales ofrecían esa noche, se destacó la posibilidad de interactuar con distintos objetos y contenidos. Una persona entrevistada señaló: [Me gustó] "Una gran variedad de opciones de muestras de arte visual y musical, mucha gente de diferentes edades y contextos sociales compartiendo espacios culturales" (E10- 2018). Otras respuestas en los cuestionarios fueron: [Me gustó] "Muestra en la calle. Muestra música" (P82- 2018), "Eventos callejeros (en la calle), baile, exposición artística" (P142017). Algunos participantes también mencionaron como positivo que el evento se realice de noche y que convoque a públicos diversos.

Melgar et al. (2017) en su investigación lograron apreciar que el público valoró de manera positiva la diversidad y la cantidad de propuestas que tienen como destinatario a un público infantil y para un público en general. Los encuestados además expresaron su opinión sobre los objetos y contenidos que conforman el patrimonio de cada museo, también se pudo apreciar que hubo personas a las que le gustaba todo del museo y, no algo en particular. Por otra parte, en esta investigación se pudo señalar la importancia que tiene para el público la realización del evento en el horario nocturno, el valor de la difusión y la convocatoria. En los estudios de Bialogorski y Fritz (2004) y Zabala y De Carli (2015) también se observó que la nocturnidad es una característica del evento que es valorada positivamente por los participantes.

Lo que ocurre es que los marcos institucionales establecen los usos posibles de los lugares en diferentes horas. Las prácticas sociales se institucionalizan espacial y temporalmente. La ciudad de noche es otra ciudad, la noche resignifica la ciudad (...). La "noche" es asociada con una predisposición anímica y una percepción de las vivencias y del tiempo, diferente a las diurnas. En términos más genéricos, la "noche" se vincula con "misterio", "magia", "romanticismo" lo cual imprime un halo de misticismo a la experiencia de recorrer ámbitos de la ciudad que no forman parte del circuito nocturno de esparcimiento habitual. (...) Se podría pensar la Noche de los Museos en el sentido antropológico de fiesta, en cuanto acontecimiento trascendente en la vida social, en la cual se subvierten el tiempo y el espacio habituales (y esto tiene que ver con la nocturnidad), y se afirman los lazos comunales. (Bialogorski y Fritz, 2004: 10-11)

Asimismo, Zabala y De Carli (2015) también destacaron la importancia del evento como oportunidad de los museos de acercase a nuevos y diversos públicos. Durante el evento es posible observar grupos etarios variados realizando recorridos con una impronta propia. En la NM es posible habitar, agasajar y crear (Melgar, Elisondo y Donolo, 2018). Habitar los museos para descubrirlos, sorprendernos, abrir sus fronteras, recorrerlos en orden o desorden, detenernos, perdernos. Agasajar a los invitados, a los públicos, disponerse para ofrecer afecto, 
atención y amabilidad. Finalmente, la NM se presenta como una instancia para crear, nuevos recorridos, nuevas experiencias, nuevas formas de experimentar el patrimonio, nuevas maneras de compartir con otros (familia, parejas, amigos y desconocidos), nuevas preguntas e interrogantes que actúen como motor para volver al año siguiente.

\section{El público propone}

En esta categoría consideramos las respuestas de los públicos en torno a los aspectos que modificarían del evento. Entre las sugerencias expresadas, se encuentran aspectos relacionados con el personal de la institución, especialmente se reconoce la necesidad de ofrecer procesos de medicación cultural y educativa, también mencionaron que sería importante ofrecer un servicio de transportes para realizar el recorrido. Además, las personas expresaron recomendaciones en relación al tiempo, el espacio físico, actividades apropiadas para niños y jóvenes, mayor incentivo y motivación por parte del museo para la participación del público, y sumar el uso de las tecnologías. Sin embargo, hay un importante número de personas que no realizarían ninguna modificación en el evento (58 personas). A modo de ejemplo algunos comentarios de los públicos: "Alguien que guíe y te cuente lo que ves" "Más dias, más jornadas, más difusión"

Teniendo en cuenta la investigación de Melgar et al. (2017) la mitad de las personas encuestadas expresaron que no modificarían nada del evento. También, los públicos indicaron su opinión respecto a la duración del evento, la forma de publicitar y difundir, los tipos de actividades que se realizan, la forma en que se organiza el evento, haciendo alusión esto último a la simultaneidad de tareas en todos los museos, el manejo del tránsito de la vía pública, entre otros aspectos. En lo que alude a la estructura y contexto del museo también se recibió sugerencia, respecto a mejorar la iluminación, contar con alguien que reciba a los visitantes, el tema de la higiene, la cartelería, etc. (Melgar et al. 2017).

En resumen, nuestra investigación y la de otros autores, nos permiten entender desde las miradas de los visitantes que, para que las experiencias sean enriquecedoras el público propone sugerencias como: la duración del evento (más días), la organización en sí, desde aspectos vinculados a la organización del tránsito en vía pública hasta la atención brindada por los organizadores del evento. No debemos olvidar que el público participante de este estudio expresó la necesidad de generar más participación por parte del público y más difusión del evento. Como se puede reflejar a continuación: "(...) que se haga más veces en el año (...)" (E9- 2018).

"Me gustaría que durará más de una noche, qué hubiera más cosas en la calle, por ahi se puede mezclar con una feria, más música, hacer mayor utilización del espacio público, hacer una guía para que uno puede ir y que durase hasta más tarde, porque creo que duraba más o menos hasta las 23:00 hs." (E4- 2017).
En el estudio de Zabala y De Carli (2015) los participantes también propusieron que el evento se desarrolle en varios días y que tenga varias ediciones en el año. Bialogorski y Fritz (2004) pudieron apreciar entre las sugerencias de los públicos, cambios referidos a la forma de organización del evento, facilitando a las personas poder llegar a la apertura de los museos o a las actividades que propone para la noche, por otra parte, expresan que sería bueno extender la duración del evento, como mayor cantidad de días.

Las expresiones de los participantes en el presente estudio y en investigaciones anteriores dan cuenta del papel activo de las personas en la apropiación de la NM. Los participantes realizan propuestas y sugerencias para el evento proyectando en nuevas ediciones.

\section{¿Aprender en la Noche de los Museos?}

En esta categoría analizamos las expresiones de los entrevistados sobre las potencialidades de la NM como experiencia de aprendizaje. ¿Es posible aprender en la NM? ¿Qué se puede aprender?

Las entrevistas nos permitieron conocer que las personas consultadas refirieron al aprendizaje como una actividad que se produce en diversidades de contextos reconociéndose al museo como uno de esos escenarios. Algunas respuestas de las entrevistas fueron: "Se aprende en cualquier lugar, hay que estar dispuesto a que suceda" (E2-2018), "En todos lados, todo el tiempo aprendemos, a veces el aprendizaje es formal otras veces cotidiano" (E10-2018). "Los museos son una oportunidad de aprender" (E22018), "Si, es un lugar muy bueno para aprender y hacerse preguntas (...)" (E3-2018).

Las expresiones de los participantes se vinculan con supuestos básicos de las perspectivas educativas socioculturales: siempre se aprende con otros, en interacción con diversos objetos y contextos. Los aprendizajes se construyen gracias a las mediaciones del lenguaje y la cultura (Bruner, 1997; Cole, 1999). En el marco de estos estudios socioculturales Rinaudo (2014) define a los contextos poderosos de aprendizaje como espacios que promueven aprendizaje activo y constructivo, presentando oportunidades para las actividades colaborativas y generalmente su experiencia de aprendizaje sucede en el marco de una situación real.

Los aprendizajes ocurren en diversos entornos de interacción cotidiana, en contextos formales, no formales e informales. Los museos pueden identificarse como contextos no formales que ponen a disposición de los públicos objetos, memorias, patrimonios, y a su vez, brindan la oportunidad de que se expresen diferentes aprendizajes (Gerber, 2001).

Tomando en cuenta la definición de Rinaudo (2014) la NM podría considerarse un contexto poderoso de aprendizaje tal como se observa en las expresiones de los entrevistados: 


\begin{abstract}
"Son espacios de disfrute, aprendizajes $e$ inspiración" (E2-2018).
\end{abstract}

\begin{abstract}
"Si, (...) claro que sí creo porque para mucha gente es la primera oportunidad, (...) hay gente que, al tener experiencias en estos contextos culturales así, también es interesante cuando el museo sale a la calle entonces ahi es como que se abre más (...)" (E5-2018).
\end{abstract}

"Para mí, lo que me facilitó aprender de los diferentes puestos, que, al ser libre, el trayecto de todos los lugares al que vos podías ir, que vos podías tomarte tu tiempo de lo que vos querías ver, interpretar. Estuvo bueno, yo creo que aprendes más así, como si fuera un paseo a que fuera guiado, es como que eso está bueno" (E6-2018).

Los museos son espacios de experiencias enriquecedoras de socialización y aprendizaje, éstos podrían promover la capacidad crítica de las personas, aumentando su interés, mejorando su capacidad para comprender el contexto social, cultural y estético, perfeccionando además así sus habilidades, destrezas y conocimientos (Pons Parra, 2016). Además, los autores Falk y Dierking (2000), plantean que los museos constituyen contextos poderosos para el aprendizaje, ya sea para complementar la educación formal o bien, porque las personas se acercan a éstos por mero interés personal.

Los visitantes de museos construyen significados a partir de sus experiencias, los intereses, los valores y las expectativas. Es por ello que, como expresa Canclini (1987 citado en Milman 2018) es importante que se lleven a cabo políticas culturales por parte de las diferentes instituciones privadas, civiles o estatales que favorezcan el desarrollo simbólico, las valoraciones, la participación, el consenso y la transformación social.

En síntesis, las actividades o elementos que facilitaron el aprendizaje fueron la participación en los diferentes talleres y exposiciones culturales, la libertad de poder recorrer los distintos lugares y tomarse el tiempo necesario para poder apreciar y comprender lo que se está visualizando, la difusión del evento. Por otro lado, las personas apreciaron de manera positiva la realización del evento la NM ya que es una forma de socializar la cultura de la ciudad, de aprender, de que lleguen a estos lugares nuevos públicos que nunca antes asistieron a los museos por diferentes razones, es un espacio de inspiración y enriquecimiento para cada persona y la comunidad.

\section{Consideraciones finales}

La NM no es un evento cultural más, es una experiencia social y educativa que invita a nuevos públicos a los museos y los espacios culturales. Es una experiencia nueva para muchos, es una invitación esperada todos los años, para otros. Los públicos se reconfiguran en la NM, algunos van siempre a los museos, otros lo hacen solo en esa noche. La mayoría de las personas consultadas en este estudio empleó medios particulares para acercarse al evento, aspecto que también indica interés para poder participar de las propuestas.

Ciertas características de la NM, nocturnidad, experiencia compartida con familiares y amigos, diversidad de actividades y lugares de encuentro, hacen que la experiencia sea significativa para los participantes, genere deseo de aprender y de participar en nuevas ediciones. La curiosidad y el deseo de aprender atraviesa toda la experiencia, tal como se ha observado en los datos y en estudios anteriores (Bialogorski y Fritz, 2004; Melgar, Elisondo y Chiecher, 2016

En la NM se aprende sobre los museos, las actividades culturales, los contenidos específicos de los museos, entre otras cuestiones. Pero tal vez lo más interesante sea que en la NM se genera una experiencia educativa compartida entre personas de diferentes edades, trayectorias e intereses culturales.

Tal como ya planteamos (Melgar, Elisondo y Chiecher, 2016), la NM es una ocasión para la creatividad porque se ofrecen posibilidades de interacción con personas, objetos culturales, narrativas e historias. La NM activa el pensamiento divergente, múltiples alternativas y recorridos son posibles, diversos lenguajes son "hablados", infinitas preguntas quedan abiertas.

Acordamos con Gordin y Dedova (2014) en que la NM también potencia la creatividad de los museos y de las personas que los gestionan. La NM supone planificaciones de actividades, recorridos y formas de interpelar a los visitantes. Asimismo, promueve la comunicación entre diferentes espacios generando novedosas redes de colaboración.

Los públicos juegan un papel activo en la NM, recorren los laberintos propuestos de diversas maneras, arman mapas originales y también generan propuestas. Más actividad, más días, más eventos, más formas de llegar, más NM en los barrios, muestran que hay mucho por hacer.

Como futuras líneas de investigación, se propone ampliar la muestra de sujetos consultados involucrando a personas que participan del evento en diferentes ciudades de Argentina y del mundo con el objetivo de comprar percepciones y experiencias de diferentes grupos. También sería interesante analizar las actividades y propuestas desarrolladas en la NM de diferentes ciudades. Una tercera línea podría considerar las experiencias y percepciones de educadores y gestores culturales en la planificación, implementación y evaluación del evento en diferentes países.

Un dato interesante para pensar en futuras líneas de acción es que, de las personas consultadas, muchos señalaron 
que llegaron al evento caminando. Este aspecto podría sugerir que estos públicos se encuentran viviendo próximos al micro y macrocentro de la ciudad donde se desarrolla la mayor parte de la NM. En este sentido, podríamos preguntarnos ¿Qué acciones, programas o políticas podrían asegurar que la NM llegue a los barrios que se encuentran alejados del centro? ¿Qué redes y con qué actores podría trabajarse para que los barrios participen en la NM?

Una segunda línea de acción vinculada a la primera tomaría en consideración resultados de estudios anteriores en los que muchos estudiantes señalan que sus primeras experiencias en museos estuvieron enmarcadas en visitas escolares ¿Cómo las escuelas de nivel medio y primario pueden involucrarse en el espacio de participación ciudadana de la NM? ¿Qué estrategias emplear para que el evento se convierta en una política que transciende una noche? ¿Qué acciones de participación ciudadana pueden gestarse para que la NM se extienda como parte del consumo cultural de otros colectivos (¿más allá de estudiantes, profesionales, comerciantes? Si entendemos a los museos como agentes de cambio social podríamos preguntarnos ¿Qué participación pueden tener grupos y temas de debate actual vinculados al género, a los sujetos en situación de discapacidad y otros grupos en el marco de la NM?

Para finalizar, son múltiples las líneas de investigación y acción que pueden generarse a partir de las percepciones y experiencias de las personas en la NM, pensamos que es una perspectiva promisoria para generar conocimientos que se acerquen a las expectativas de los públicos que van a los museos.

\section{Agradecimientos}

A la Agencia Nacional de Promoción Científica y Tecnológica (ANPCYT) que financió el PICT 2789/17 denominado EDUCACIÓN Y APRENDIZAJE EN MUSEOS Estudios de experiencias museísticas en entornos virtuales y físicos que permitió la realización del Trabajo Final de Licenciatura en Psicopedagogía Aprendizaje y Percepciones en la Noche de los Museos.

\section{Referencias Bibliográficas}

Barron, P y Leask, A (2017) Visitor engagement at museums: Generation $Y$ and 'Lates' events at the National Museum of Scotland. Museum Management and Curatorship, 32:5, 473490, DOI: 10.1080/09647775.2017.1367259

Bialogorski, M. y Fritz, P (2004), “CCómo impacta la Noche de los Museos en nuestros visitantes? Un estudio de público en la ciudad de Buenos Aires". Inédito. Recuperado de http:// network.icom.museum/fileadmin/user_upload/minisites/icomargentina/pdf/BIALOGORSKI-FRITZ_NOCHE_DE_LOS_MUSEOS. pdf- consultado el día 18-09-2019.

Bruner, J. (1997). La Educación, puerta de la cultura. Barcelona: Aprendizaje Visor
Cole, M. (1999). Psicología Cultural. Una disciplina del pasado y del futuro. Madrid: Morata.

Elas, S. y Leonardi, V. (2018), La noche de los museos en Buenos Aires en el marco del turismo cultural. Recuperado de https:// dialnet.unirioja.es/servlet/articulo?codigo $=6358786$ Consultado el día 22-03-19.

Falk, J. y Dierking, L. (2000), Learning from museums. Visitor experiences and the making of meaning. Lanham, USA: Altamira Press.

Gerber, G.L. (2001), Relationships among informal learning environments, teaching procedures and scientific reasoning ability. International Journal of Science Education, 23(5), 535549.

Germain. F. (2016) Visiting the museum at night: A decidedly different experience. Loisir et Société/Society and Leisure, 39:3, 433-450. DOI: 10.1080/07053436.2016.1244146.

Gordin, V., y Dedova, M. (2014). Cultural innovations and consumer behaviour: the case of Museum Night. International Journal of Management Cases, 16 (2): 32-40.

Melgar, M. F; Elisondo, R y Donolo, D (2018). Experiencias en museos. Zonas educativas posibles. Revista Edetania. Estudios y propuestas socioeducativas, 53: 241-256. Recuperado de: http://revistas.ucv.es/index.php/Edetania/article/view/333/360. Consultado 23/4/2020

Melgar, M.F., Chiecher, A., Elisondo, R., Donolo, D. (2017), "Museos y consumo cultural. Percepciones y experiencias en la Noche de los Museos". E-rph: Revista electrónica de Patrimonio Histórico, ISSN-e 1988-7213, №. 21, 2017, págs. 171-204. Recuperado de https://revistadepatrimonio.es/index.php/erph/ article/download/243/227 consultado el día 11-09-2019.

Melgar, M.F., Elisondo, R., Chiecher, A. (2016), Ciudades, museos y creatividad. Estudio de público en la Noche de los Museos. Arte y Ciudad - Revista de Investigación No 10 octubre de 2016. Recuperado de https://dialnet.unirioja.es > descarga > articulo consultado el día 19-09-2019.

Milman, E. (2018), "Significaciones en torno a la "Noche de los Museos" en el Museo de Antropología de la Universidad Nacional de Córdoba".Trabajo Inédito. Universidad Nacional de Córdoba.

Rinaudo, M. (2014), Estudios sobre los contextos de aprendizaje. Arenas y fronteras. En P. Paoloni, M. Rinaudo\& A. González Fernández (comps.) Cuestiones en Psicología Educacional: cuestiones teóricas, metodológicas y estudios de campo (pp.163-205). Tenerife: Sociedad Latinoamericana de Comunicación Social (SLCS). Recuperado de http://www. cuadernosartesanos.org/2014/cde01.pdf. Consultado el 3008-2019.

Zabala, M.E. y De Carli, M.C. (2015), La "noche" construida por los visitantes del Museo de Antropología FFyH- UNC. Caso de estudio, la Noche de los Museos, Revista del Museo de Antropología, 8 (1): 125-132. Recuperado de https://revistas. unc.edu.ar/index.php/antropologia/article/view/11465/12174 Consultado el día 18-09-2019. 